\title{
TRANSMISSION EIGENVALUES
}

\author{
LASSI PÄIVÄRINTA * AND JOHN SYLVESTER †
}

\begin{abstract}
The scattering of a time-harmonic plane wave in an inhomogeneous medium is modeled by the scattering problem for the Helmholtz equation. A transmission eigenvalue is a wavenumber at which the scattering operator has a non-trivial kernel or cokernel. Because many sampling methods for locating scatterers succeed only at wavenumbers that are not transmission eigenvalues, they have been studied for some time. Nevertheless, the existence of transmission eigenvalues has previously been proved only for radial scatterers. In this paper, we prove existence for scatterers without radial symmetry.
\end{abstract}

1. Introduction. The scattering of a time-harmonic plane wave in an inhomogeneous medium is modeled by the scattering problem for the Helmholtz equation. The total wave $u$ satisfies the perturbed Helmholtz equation

$$
\left(\Delta+k^{2}(1+m)\right) u=0 \quad \text { in } \mathbb{R}^{n}
$$

The function $m(x)$ denotes the perturbation of the index of refraction from the constant background medium; i.e. $n^{2}(x)=1+m(x)$. We insist that $-1<m(x)$, be compactly supported and bounded. The relative (far field) scattering operator, $s^{+}$, compares the asymptotics of solutions of the free Helmholtz equation to those of (1.1). Both the linear sampling method and the factorization method use the range of this operator to find the support of the scatterer $m$. These methods are known to succeed at wavenumbers $k$ for which the range of that operator is dense among all far field patterns (i.e. dense in $L^{2}\left(S^{n-1}\right)$ ). If there exists a bounded domain $D$ that contains the support of $m(x)$, and the wavenumber $k$ is not a transmission eigenvalue as defined below, then the range of the scattering operator is dense. [5].

Definition 1.1. A wavenumber $k$ is called a transmission eigenvalue if there exists a non-trivial pair $(v, w)$ solving

$$
\begin{aligned}
& \Delta w+k^{2} n^{2}(x) w=0 \quad \text { in } \quad D \\
& \Delta v+k^{2} v=0 \quad \text { in } \quad D \\
& w=v, \frac{\partial w}{\partial \nu}=\frac{\partial v}{\partial \nu} \quad \text { on } \quad \partial D
\end{aligned}
$$

If $D$ is not smooth enough, we replace (1.4) with the condition that $u-v \in H_{0}^{2}(D)$. Under the conditions that $m>0$ or $m<0$ on its support, it has been shown that the set of transmission eigenvalue is at most discrete [4], [12], but existence has only been established for $m$ which depend only on the radius, [6]. Under certain conditions, knowledge of the transmission eigenvalues uniquely determines a radial scatterer [9] [10]. For non-radial scatterers, transmission eigenvalues have also been used to infer simple properties of the scatterer [3].

Under the hypothesis that the infimum of $|m|$ is large enough, we prove existence of transmission eigenvalues, as well as upper and lower bounds on the first transmission eigenvalue. The existence and upper bounds are new; the lower bounds are

\footnotetext{
*Department of Mathematics and Statistics, University of Helsinki, Helsinki, Finland, Research supported by a grant from the Academy of Finland (ljp@rni.helsinki.fi)

$\dagger$ Department of Mathematics, University of Washington, Seattle, Washington 98195, Research supported by ONR grant N00014-05-1-0716 and NSF grant DMS-0355455 (sylvest@u.washington.edu)
} 
results from [7] and [3].

In [7], we showed that the following three conditions were equivalent. Notice that (1.5) differs from (1.3) in that the condition below requires that $v$ solve the free Helmholtz equation in all or $\mathbb{R}^{n}$ rather than just in $D$. Such $v$ which can be represented as superpositions of plane waves with $L^{2}$ densities are called Herglotz wave-functions.

1. There exists a non-trivial pair $(v, w)$ solving

$$
\begin{aligned}
& \Delta w+k^{2} n^{2}(x) w=0 \quad \text { in } \quad D \\
& \Delta v+k^{2} v=0 \quad \text { in } \quad \mathbb{R}^{n} \\
& w=v, \frac{\partial w}{\partial \nu}=\frac{\partial v}{\partial \nu} \quad \text { on } \quad \partial D
\end{aligned}
$$

2. There exists a nontrivial $\mu^{0} \in \operatorname{ker} s^{+}$

3. There exists a nontrivial $\mu^{0} \in$ coker $s^{+}$

In the case that $v$ is a Herglotz wave-function, its asymptotic expansion (its far field) belongs to both the kernel and the cokernel of the far field scattering operator. We will show below that, for scatterers supported in a compact set $D$, the far field scattering operator has a natural extension, and that transmission eigenvalues are exactly the wavenumbers for which this natural extension has a kernel or cokernel.

2. The Helmholtz Equation and the Scattering Operator. The scattering operator relates the solutions of (1.1) to solutions of the free Helmholtz equation in all of $\mathbb{R}^{n}$.

$$
\left(\Delta+k^{2}\right) u^{0}=0 \quad \text { in } \mathbb{R}^{n}
$$

We refer to solutions of (2.1) with finite $B^{*}$-norm, defined by:

$$
\left\|u^{0}\right\|_{B^{*}}=\sup _{R>0} \frac{1}{\sqrt{R}}\left\|u^{0}\right\|_{L^{2}\left(B_{R}\right)}
$$

as incident waves or free waves. An outgoing wave is a solution to the Helmholtz equation with a compactly supported source $f$.

$$
\left(\Delta+k^{2}\right) v^{+}=f \quad \text { in } \mathbb{R}^{n}
$$

that satisfies the Sommerfeld radiation condition

$$
\lim _{r \rightarrow \infty} r^{\frac{n-1}{2}}\left(\frac{\partial v^{+}}{\partial r}-i k v^{+}\right)=0
$$

or equivalently (for $k>0$ ), a limiting absorption principle

$$
v^{+}=\lim _{\varepsilon \downarrow 0} v_{\varepsilon}^{+}
$$

where $v_{\varepsilon}^{+}$is the unique solution to $(2.2)$ with $k^{2} \in \mathbb{R}$ replaced by $k^{2}+i \varepsilon$ (see e.g. section 4 of [1]). We could also define an outgoing wave as a solution to

$$
\left(\Delta+k^{2}(1+m)\right) w^{+}=g
$$

with a compactly supported $g$, and satisfying (2.3) or (2.4). Because $m$ is compactly supported, the definition based on (2.5) and that based on (2.2) coincide. That is, an 
outgoing solution $v^{+}$to $(2.2)$ is also an outgoing solution $w^{+}$to $(2.5)$ with $g=f-m v^{+}$.

Existence and uniqueness of outgoing solutions to (2.5) was proved by Agmon, in weighted $L^{2}$ spaces ${ }^{1}$ [1]. Theorem 2.1 below is a special case of results in [2] and parts of theorems 2.2 and 2.3 are special cases of results in [2] and section 14 of [8].

Define $B$ to be the completion of $C_{0}^{\infty}\left(\mathbb{R}^{n}\right)$ in the $B$ norm

$$
\|f\|_{B}=\|f\|_{L^{2}(|x| \in[0,1])}+\sum_{j=1}^{\infty} \frac{1}{\sqrt{2^{j}}}\|f\|_{L^{2}\left(|x| \in\left[2^{j}, 2^{j+1}\right]\right)}
$$

ThEOREM 2.1. For every compactly supported $g$, there exists a unique outgoing solution to (2.5), with

$$
\left\|w^{+}\right\|_{B^{*}} \leq C\|g\|_{B}
$$

where the constant $C$ depends on $m$ and $k$.

Because compactly supported functions are dense in $B$, the correspondence in theorem 2.1 defines a bounded map

$$
G_{m}^{+}: B \longrightarrow B^{*}
$$

mapping $g \in B$ to $w^{+}$. In the rest of the paper, whenever we refer to waves, we mean subspaces of $B^{*}$.

1. $B^{0}$ is the subspace of incident waves, i.e. solutions to (2.1).

2. $B^{m}$ is the subspace of total waves, i.e. solutions to (1.1).

3. $B^{+}$is the subspace of outgoing waves, the range of $G_{0}^{+} \cdot{ }^{2}$

Both $B^{0}$ and $B^{m}$ are closed in the $B^{*}$ topology. One way to see this is to note that $B^{*}$ convergence implies convergence in the sense of tempered distributions, so that any $u$ in the closure of $B^{0}$ or $B^{m}$ must satisfy (2.1) or (1.1), respectively, in the sense of distributions. As Schwartz class functions are dense in $B$, the equations are satisfied in the $B^{*}$ sense as well. The plane waves, $e^{i k \Theta \cdot x}$, are not in $B^{0}$. We shall note in theorem 2.3 below that $B^{0}$ consists of the Herglotz wave functions, solutions to (2.1) which have square integrable far fields. The far fields of the plane waves are Dirac deltas. The subspace $B^{+}$is not closed in the $B^{*}$ topology. In particular, every function in $B^{+}$is in $H_{l o c}^{2}\left(\mathbb{R}^{n}\right)$ and satisfies the radiation condition (2.3), and every compactly supported function in $H_{l o c}^{2}\left(\mathbb{R}^{n}\right)$ belongs to $B^{+}$. Because we have defined $B^{+}$as the range of $G_{0}^{+}$, which is injective, $B^{+}$is a Banach space with norm $\left\|G_{0}^{+} f\right\|_{B^{+}}:=\|f\|_{B}$.

A straightforward consequence of theorem 2.1 is the correspondence between incident and total waves.

THEOREM 2.2. Every total wave has a unique decomposition into an incident wave plus a scattered wave, and every incident wave has a unique decomposition as a total wave minus a scattered wave.

$$
\begin{aligned}
v^{m} & =v^{0}+v^{+} \\
u^{0} & =u^{m}-u^{+}
\end{aligned}
$$

\footnotetext{
${ }^{1}\|f\|_{L_{\delta}^{2}}=\left\|\left(1+|x|^{2}\right)^{\frac{\delta}{2}} f\right\|_{L^{2}}$

${ }^{2}$ which is also the range of $G_{m}^{+}$, for any bounded compactly supported $m$.
} 
Moreover, the scattering map $\mathcal{S}$, defined as $u^{0} \mapsto u^{m}$ is an isomorphism from $B^{0}$ onto $B^{m}$,

Proof. We prove the second assertion first. Any $u_{0}$ that solves (2.1) also solves

$$
\left(\Delta+k^{2}(1+m)\right) u^{0}=k^{2} m u^{0}
$$

Let $u^{+}$be the unique outgoing solution to (2.5) with $g=-k^{2} m u^{0}$. Note that,

$$
\left\|u^{+}\right\|_{B^{*}} \leq C_{1}\|g\|_{B} \leq k^{2} C_{1}\left\|m u^{0}\right\|_{B} \leq k^{2} C_{2}\left\|u^{0}\right\|_{B^{*}}
$$

where both the constants depend on an upper bound for $m$ and the size of its support.

Defining $u^{m}=u^{0}+u^{+}$and noting that it satisfies (1.1) gives a decomposition (2.7), and the estimate

$$
\left\|u^{m}\right\|_{B^{*}} \leq C_{3}\left\|u^{0}\right\|_{B^{*}}
$$

If $u^{0}=w^{m}-w^{+}$is another such decomposition, then $w^{+}$must also satisfy (2.5) with $g=-k^{2} m u^{0}$, but (2.5) has a unique outgoing solution, so $w^{+}=u^{+}$and $w^{m}=u^{m}$.

Similarly, any $v^{m}$ solving (1.1) is a solution to

$$
\left(\Delta+k^{2}\right) v^{m}=-k^{2} m v^{m}
$$

Let $v^{+}$be the unique outgoing solution to (2.2) with $f=-k^{2} m u^{m}$ and set $v^{0}=$ $v^{m}-v^{+}$. Uniqueness follows as in the paragraph above, as does the estimate

$$
\left\|u^{0}\right\|_{B^{*}} \leq C_{3}\left\|u^{m}\right\|_{B^{*}}
$$

The existence and uniqueness of the two decompositions, (2.6) and (2.7), along with the estimates, (2.8) and (2.9), justify the last statement in the theorem, that the scattering map is an isomorphism.

In order to see the relationship between the scattering operator we have defined above, and the scattering operator defined on far fields, we need to discuss asymptotics.

Theorem 2.3. Let $u^{0} \in B^{0}, u^{+} \in B^{+}$, and $u^{m} \in B^{m}$, then, in spherical coordinates $x=r \Theta$, for large $r$,

$$
\begin{aligned}
& u^{0} \sim \mu^{0}(\Theta) \frac{e^{i k r}}{(i k r)^{\frac{n-1}{2}}}+\mu^{0}(-\Theta) \frac{e^{-i k r}}{(-i k r)^{\frac{n-1}{2}}} \\
& u^{+} \sim \mu^{+}(\Theta) \frac{e^{i k r}}{(i k r)^{\frac{n-1}{2}}} \\
& u^{m} \sim\left(\mu^{m}(\Theta)+\gamma(\Theta)\right) \frac{e^{i k r}}{(i k r)^{\frac{n-1}{2}}}+\mu^{m}(-\Theta) \frac{e^{-i k r}}{(-i k r)^{\frac{n-1}{2}}}
\end{aligned}
$$

Moreover, the mappings

$$
\begin{array}{r}
b^{0}: B^{0} \longrightarrow L^{2}\left(S^{n-1}\right) \\
b^{m}: B^{m} \longrightarrow L^{2}\left(S^{n-1}\right)
\end{array}
$$


defined by $u^{0} \mapsto \mu^{0}$ and by $u^{m} \mapsto \mu^{m}$ are isomorphisms. The mapping

$$
b^{+}: B^{+} \longrightarrow L^{2}\left(S^{n-1}\right)
$$

defined by $u^{+} \mapsto \mu^{+}$is surjective ${ }^{3}$. Every compactly supported function in $H^{2}\left(\mathbb{R}^{n}\right)$ belongs to its kernel and any function in its kernel is compactly supported (Rellich's Lemma).

Proof. [Sketch of Proof] The operator $\left(b^{0}\right)^{-1}$ is known as the Herglotz operator. We can start with any $\mu_{0} \in L^{2}\left(S^{n-1}\right)$ and define:

$$
u^{0}=\mathcal{H} \mu^{0}=\int_{S^{n-1}} e^{i k \Theta \cdot x} \mu^{0}(\Theta) d S_{\Theta}
$$

Noting that any $u^{0} \in B^{0}$ is the inverse Fourier transform of a distribution supported on the sphere $|\xi|^{2}-k^{2}=0$ shows that $u^{0}$ must have this form, but it requires an estimate [2] to see that $\mu^{0} \in L^{2}$. A stationary phase calculation shows that $\mathcal{H} \mu^{0}$ has the asymptotics (2.10) when $\mu^{0}$ is smooth. Again we refer to [2] for the estimate that $\|u\|_{B^{*}} \leq C\left\|\mu^{0}\right\|_{L^{2}\left(S^{n-1}\right)}$.

A similar Fourier transform calculation combined with the limiting absorption principle, or a calculation of the asymptotics of the outgoing Green's function gives (2.11). Alternatively, we may note that $u^{+}(x, k)-u^{+}(x,-k)^{4}$ belongs to $B^{0}$, and deduce (2.11) from (2.10). The surjectivity of $b^{+}$then follows from the surjectivity of $b^{0}$.

Because of the decomposition $u^{m}=u^{0}+u^{+}$in theorem 2.2, (2.12) follows from (2.10) and (2.11). Rellich's lemma and unique continuation imply that $b^{0}$ and $b^{m}$ are injective, as well as the final statement in the theorem.

We refer to the large $r$ asymptotics as the far fields of the corresponding waves, e.g. the far field of $u^{0}$ is $\mu^{0}$, the far field of $u^{+}$is $\mu^{+}$, and the far field of $u^{m}$ is $\mu^{m}$. We use (2.12) to define the far field (relative scattering) operator.

$$
s^{+}: L^{2}\left(S^{n-1}\right) \longrightarrow L^{2}\left(S^{n-1}\right)
$$

by

$$
\mu^{m} \mapsto \gamma
$$

The wave scattering operator $\mathcal{S}$ and the far field operator $s^{+}$are closely related. Lemma 2.4. Let $u^{0}$ and $w^{0}$ belong to $B^{0}$, with far fields $\mu^{0}$ and $\omega^{0}$, respectively. Then

$$
\int_{\mathbb{R}^{n}} \overline{u^{0}} m \mathcal{S} w^{0}=\frac{-2 i}{k^{n}} \int_{S^{n-1}} \overline{\mu^{0}} s^{+} \omega^{0}
$$

\footnotetext{
${ }^{3}$ The surjectivity of $b^{+}, b^{0}$, and $b^{m}$, is perhaps the main reason for replacing the $L_{\delta}^{2}$ spaces in [1] with the Besov spaces, $B$ and $B^{*}$, of [2].

${ }^{4}$ Changing the sign of $k$ reverses the sign of $\epsilon$ in the limiting absorption principle and changes the sign of the second term in the Sommerfeld radiation condition, thus specifying the unique incoming, rather than the outgoing solution.
} 
Proof.

$$
\int_{\mathbb{R}^{n}} \overline{u^{0}} k^{2} m \mathcal{S} w^{0}=\int_{\mathbb{R}^{n}} \overline{u^{0}} k^{2} m w^{m}
$$

where $w^{m}=w^{0}+w^{+}$, as in $(2.6)$

$$
\begin{aligned}
& =-\int_{\mathbb{R}^{n}} \overline{u^{0}}\left(\Delta+k^{2}\right) w^{m} \\
& =-\int_{\mathbb{R}^{n}} \overline{u^{0}}\left(\Delta+k^{2}\right) w^{+} \\
& =-\lim _{R \rightarrow \infty} \int_{|x|<R} \overline{u^{0}}\left(\Delta+k^{2}\right) w^{+} \\
& =\lim _{R \rightarrow \infty} \int_{|x|=R} \frac{\partial \overline{u^{0}}}{\partial \nu} w^{+}-\overline{u^{0}} \frac{\partial w^{+}}{\partial \nu}
\end{aligned}
$$

Making use of the asymptotics in (2.11) and (2.12) gives:

$$
=\frac{-2 i}{k^{n-2}} \int_{S^{n-1}} \overline{\mu^{0}} \omega^{+}
$$

where $\omega^{+}$denotes the far field of $w^{+}$.

$$
=\frac{-2 i}{k^{n-2}} \int_{S^{n-1}} \overline{\mu^{0}} s^{+} \omega^{0}
$$

$\square$

A consequence of lemma 2.4 is a natural definition of the relative scattering operator which doesn't explicitly use asymptotics.

ThEOREM 2.5. If we define

$$
\mathcal{S}^{+}: B^{0} \longrightarrow B^{0^{*}}
$$

by

$$
w^{0} \stackrel{\mathcal{S}^{+}}{\longmapsto} \frac{-k^{n}}{2 i}\left\langle m \mathcal{S} w^{0}, \cdot\right\rangle
$$

then

$$
s^{+}=\mathcal{H}^{*} \mathcal{S}^{+} \mathcal{H}
$$


Proof.

$$
\begin{aligned}
\left\langle\mathcal{H}^{*} \mathcal{S}^{+} \mathcal{H} \omega^{0}, \overline{\mu^{0}}\right\rangle & =\left\langle\mathcal{S}^{+} \mathcal{H} \omega^{0}, \overline{\mathcal{H} \mu^{0}}\right\rangle \\
& =\frac{-k^{n}}{2 i} \int_{\mathbb{R}^{n}} \overline{\mathcal{H} \mu^{0}} m \mathcal{S} \mathcal{H} \omega^{0} \\
& =\frac{-k^{n}}{2 i} \int_{\mathbb{R}^{n}} \overline{u^{0}} m \mathcal{S} w^{0} \\
& =\int_{S^{n-1}} \overline{\mu^{0}} s^{+} \omega^{0} \\
& =\left\langle s^{+} \omega^{0}, \overline{\mu^{0}}\right\rangle
\end{aligned}
$$

Remark 2.6. Because $B^{0} \subset L_{-\delta}^{2}$ for any $\delta>\frac{1}{2}$, any $l \in B^{0^{*}}$ has a (non-unique) extension to an element of $L_{\delta}^{2}=L_{-\delta}^{2}{ }^{*}$, so elements of $B^{0^{*}}$ can be represented as functions (and called sources).

3. A Generalized Scattering Operator. We describe an incident wave $\mathcal{H} \alpha$ as illuminating the scatterer $m$. If we use the far field operator $s^{+}$, the illumination must always come from the sphere at infinity. Many useful sources of illumination are generated by sources outside the scatterer. The waves generated by such sources are never incident waves, although they can be approximated by incident waves on certain compact sets. Solutions to the transmission eigenvalue problem, (1.2)- (1.4), are not incident waves, so they do not have a direct interpretation in terms of the far field scattering operator. They do, however, span exactly the kernel of the scattering operator we will define below.

If $m \in L^{\infty}$ is supported in a bounded domain $D$, Theorem 2.1 tells us that we can find a unique $u^{+} \in B^{+}$solving

$$
\left(\Delta+k^{2}(1+m)\right) u^{+}=k^{2} m u^{0}
$$

for any $u^{0} \in L^{2}(D)$. It follows that $u^{m}=u^{0}+u^{+} \in L^{2}(D)$. Thus the scattering operator $\mathcal{S}$ has a natural extension:

$$
\mathcal{S}_{D}: B_{D}^{0} \longrightarrow B_{D}^{m}
$$

where we use the definitions:

$$
\begin{aligned}
& B_{D}^{0}=\left\{w \in L^{2}(D) \mid\left(\Delta+k^{2}\right) w=0 \text { in } D\right\} \\
& B_{D}^{m}=\left\{w \in L^{2}(D) \mid\left(\Delta+k^{2}(1+m)\right) w=0 \text { in } D\right\}
\end{aligned}
$$

The relative scattering operator $\mathcal{S}^{+}$has a similar extension.

$$
\begin{aligned}
\mathcal{S}_{D}^{+}: & B_{D}^{0} \longrightarrow B_{D}^{0}{ }^{*} \\
w^{0} & \stackrel{\mathcal{S}_{D}^{+}}{\longmapsto} \frac{k^{n}}{2 i}\left\langle m \mathcal{S}_{D} w^{0}, \cdot\right\rangle
\end{aligned}
$$

The scattering and relative scattering operators $\mathcal{S}_{D}$ and $\mathcal{S}_{D}^{+}$are extensions of $\mathcal{S}$ and $\mathcal{S}^{+}$in the sense that, for $u^{0}, w^{0} \in B^{0}$, then $\mathcal{S}_{D} u^{0}$ is the restriction of $\mathcal{S} u^{0}$ to $D$ and $\left\langle\mathcal{S}_{D}^{+} u^{0}, w^{0}\right\rangle=\left\langle\mathcal{S}^{+} u^{0}, w^{0}\right\rangle$. 
4. The Interior Transmission Problem. Let $D$ be a bounded domain. and supp $m \subset D$. We will use the notation

$$
\begin{aligned}
P^{0} & =\left(\Delta+k^{2}\right) \\
P^{m} & =\left(\Delta+k^{2}(1+m)\right) \\
H^{k}(D) & =\left\{u \in L^{2}(D)\left|D^{\alpha} u \in L^{2}(D) \quad \forall\right| \alpha \mid \leq k\right\} \\
H_{0}^{k}(D) & =\text { the completion of } C_{0}^{\infty}(D) \text { in } H^{k}(D)
\end{aligned}
$$

DEFINITION 4.1. We say that a wavenumber $k$ is a D-transmission eigenvalue of $m \in L^{\infty}(D)$ if any of the equivalent conditions in theorem 4.2 below are satisfied.

THEOREM 4.2. The following are equivalent:

1. There exists nontrivial $u^{0} \in B_{D}^{0}$ and $u^{m} \in B_{D}^{m}$ with $u^{0}-u^{m} \in H_{0}^{2}(D) .^{5}$

2. There exists nontrivial $u^{m} \in B_{D}^{m}$ such that the unique outgoing solution $u^{+}$ to

$$
P^{0} u^{+}=-k^{2} m u^{m}
$$

belongs to $H_{0}^{2}(D)$.

3. There exists nontrivial $u^{m} \in B_{D}^{m}$ and some $v \in H_{0}^{2}(D)$ satisfying (4.1).

4. There exists nontrivial $u^{0} \in B_{D}^{0}$ such that the unique outgoing solution $u^{+}$to

$$
P^{m} u^{+}=-k^{2} m u^{0}
$$

belongs to $H_{0}^{2}(D)$.

5. There exists nontrivial $u^{0} \in B_{D}^{0}$ and some $v \in H_{0}^{2}(D)$ satisfying (4.2).

6. There exists nontrivial $u^{0} \in \operatorname{ker} \mathcal{S}_{D}^{+}$

7. There exists nontrivial $u^{0} \in$ coker $\mathcal{S}_{D}^{+}$

Proof. We first show that items $1-5$ are equivalent.

Condition 2 obviously implies 3 , but any $H_{0}^{2}(D)$ solution, $v$, to (4.1) extended to be zero in $\mathbb{R}^{n} \backslash D$, is outgoing. Since the outgoing solution to (4.1) is unique, $v=u^{+}$, so 3 implies 2 .

Similarly, 4 obviously implies 5, but uniqueness of the outgoing solution to (4.2), implies that any $H_{0}^{2}(D)$ solution to (4.2), extended to be zero outside $D$, must be $u^{+}$, so 5 implies 4 .

Because Theorem 2.2 gives a unique decomposition,

$$
u^{m}=u^{0}+u^{+}
$$

the unique outgoing solution to (4.1) is also the unique outgoing solution to (4.2). Thus 4 and 2 are equivalent.

The same decomposition shows that $u^{+}=u^{m}-u^{0}$ so the left hand side is in $H_{0}^{2}(D)$ if and only if the right hand side is; hence 1 is equivalent to 2 .

\footnotetext{
${ }^{5}$ This is a restatement of (1.2)-(1.4). The condition that $u^{0} \in B_{D}^{0}$ is (1.3), $u^{m} \in B_{D}^{m}$ is (1.2), and $u^{0}-u^{m} \in H_{0}^{2}(D)$ is (1.4)
} 
The equivalence of items 6 and 2 is based on a calculation. Let $u^{0}$ and $w_{0}$ belong to $B_{D}^{0}$.

$$
\begin{aligned}
\left\langle\overline{w^{0}}, \mathcal{S}_{D}^{+} u^{0}\right\rangle & =-\frac{k^{n}}{2 i}\left\langle\overline{w^{0}}, m \mathcal{S}_{D} u^{0}\right\rangle \\
& =-\frac{k^{n}}{2 i} \int_{D} \overline{w^{0}} m u^{m}
\end{aligned}
$$

where $u^{m}$ in the line above and $u^{+}$in the line below are those uniquely related to $u^{0}$ by (4.3) and theorem 2.2 .

$$
\begin{aligned}
& =-\frac{k^{n-2}}{2 i} \int_{D} \overline{w^{0}} P^{0} u^{+} \\
& =\frac{k^{n-2}}{2 i} \int_{\partial D} \frac{\partial \overline{w^{0}}}{\partial \nu} u^{+}-\overline{w^{0}} \frac{\partial u^{+}}{\partial \nu}
\end{aligned}
$$

The right hand side of (4.6) is clearly zero for every $w^{0}$ if $u^{+} \in H_{0}^{2}(D)$, so 2 implies 6. To see the converse, choose $w^{0}=\mathcal{H} \omega^{0}$ (i.e. $w^{0} \in B^{0}$ with asymptotics as in (2.10). Every $u^{+} \in B^{+}$has asymptotics as in (2.11), so we may continue the previous calculation,

$$
\begin{aligned}
\left\langle\overline{w^{0}}, \mathcal{S}_{D}^{+} u^{0}\right\rangle & =\frac{k^{n-2}}{2 i} \lim _{R \rightarrow \infty} \int_{B_{R}} \frac{\partial \overline{w^{0}}}{\partial \nu} u^{+}-\overline{w^{0}} \frac{\partial u^{+}}{\partial \nu} \\
& =\int_{S^{n-1}} \overline{\omega^{0}} \mu^{+}
\end{aligned}
$$

We conclude that, if the left hand side vanishes for every $w^{0}$, so does the right hand side for every $\omega^{0}$, so $\mu^{+} \equiv 0$. Now Rellich's lemma and unique continuation tell us that $u^{+} \in H_{0}^{2}(D)$, so 6 implies 2 .

Verifying he equivalence of items 7 and 2 requires a similar computation.

$$
\begin{aligned}
\frac{2 i}{k^{n-2}}\left\langle u^{0}, \mathcal{S}_{D}^{+} w^{0}\right\rangle & =-\int_{D} u^{0} k^{2} m w^{m} \\
& =\int_{D} u^{0} P^{0} w^{m} \\
& =\int_{\partial D} \frac{\partial u^{0}}{\partial \nu} w^{m}-u^{0} \frac{\partial w^{m}}{\partial \nu} \\
& =\int_{\partial D} \frac{\partial u^{0}}{\partial \nu} w^{m}-u^{0} \frac{\partial w^{m}}{\partial \nu}-\int_{\partial D} \frac{\partial u^{m}}{\partial \nu} w^{m}-u^{m} \frac{\partial w^{m}}{\partial \nu}
\end{aligned}
$$

because the second integral on the right is always zero. Combining the two terms 
gives

$$
\frac{2 i}{k^{n-2}}\left\langle u^{0}, \mathcal{S}_{D}^{+} w^{0}\right\rangle=-\int_{\partial D} \frac{\partial u^{+}}{\partial \nu} w^{m}-u^{+} \frac{\partial w^{m}}{\partial \nu}
$$

Now 2 implies that the right hand side of (4.7) is zero, so the left hand side is zero for every $w^{0}$, which implies 7 . If we choose $w^{0} \in B^{0}$ and continue the calculation:

$$
=\int_{S^{n-1}} \mu^{+} \omega^{m}
$$

Item 7 implies that the integral in (4.8) vanishes for every $\omega^{m}$, so $\mu^{+} \equiv 0$. Rellich's lemma and unique continuation then guarantee that $u^{+} \in H_{0}^{2}(D)$, which implies item 2. $\square$

Note that if, $\operatorname{supp} m \subset \tilde{D} \subset D$, then $\mathcal{S}_{\tilde{D}}$ is an extension of $\mathcal{S}_{D}$. As the domain gets smaller, $B_{D}^{0}$, the domain of $\mathcal{S}_{D}$ gets larger. Therefore, if $k$ is a $D$-transmission eigenvalue of $m$, then $k$ is also a $\tilde{D}$-transmission eigenvalue.

5. Existence of Transmission Eigenvalues. In this section we restrict to the case that $D=\operatorname{supp} m$. We assume further that $m$ is bounded away from zero in $D$. The theorem below was first proved in [12].

THEOREM 5.1. If $|m|>\delta>0$ in $D$, then $k$ is a D-transmission eigenvalue if and only if there exists $u^{+} \in H_{0}^{2}(D)$ satisfying

$$
P^{m} \frac{1}{m} P^{0} u^{+}=0
$$

Proof. We show that (5.1) is equivalent to item 2 in Theorem 4.2. If $u^{+} \in H_{0}^{2}(D)$ satisfies

$$
P^{0} u^{+}=-k^{2} m u^{m}
$$

then

and

$$
\frac{1}{m} P^{0} u^{+}=-k^{2} u^{m}
$$

$$
P^{m} \frac{1}{m} P^{0} u^{+}=0
$$

To see the reverse implication, suppose that $u^{+} \in H_{0}^{2}(D)$ satisfies (5.1) (recall that any $u^{+} \in H_{0}^{2}(D)$ is outgoing), and define $u^{m}$ so that (5.3) holds. It is a consequence of (5.1) that $u^{m} \in B_{D}^{m}$, so that (5.2) implies (4.1). $\square$

Theorem 5.1 tells us that $k$ is a $D$-transmission eigenvalue whenever the operator $P^{m} \frac{1}{m} P^{0}$ has a kernel in $H_{0}^{2}(D)$. We will investigate the existence of this kernel (5.1) by examining the spectrum of the operator as $k^{2}$ changes. We will make use of several equivalent formulas for $P^{m} \frac{1}{m} P^{0}$ which we list below. We will let $\tau=k^{2}$.

$$
\begin{aligned}
P^{m} \frac{1}{m} P^{0} & =P^{0} \frac{1}{m} P^{m} \\
& =\Delta \frac{1}{m} \Delta+\tau\left(\Delta \frac{1}{m}+\left(1+\frac{1}{m}\right) \Delta\right)+\tau^{2}\left(1+\frac{1}{m}\right) \\
& =(\Delta+\tau) \frac{1}{m}(\Delta+\tau)+\tau(\Delta+\tau) \\
& =(\Delta+\tau(1+m)) \frac{1}{m}(\Delta+\tau(1+m))-\tau(\Delta+\tau(1+m))
\end{aligned}
$$


The following lemma asserts that $P^{m} \frac{1}{m} P^{0}$, with the appropriate domain, defines a semi-bounded self-adjoint operator on $L^{2}(D)$.

LEMMA 5.2.

For $\tau \geq 0, t_{\tau}$, defined by

$$
t_{\tau}(u)=\int_{D} \frac{1}{m}|(\Delta+\tau) u|^{2}-\tau \int_{D}|\operatorname{grad} u|^{2}+\tau^{2} \int_{D}|u|^{2}
$$

with form domain $H_{0}^{2}(D)$, is a densely defined, closed semi-bounded quadratic form on $L^{2}(D) . T_{\tau}$, The unique densely defined self-adjoint operator associated to $t_{\tau}, T_{\tau}$, is equal to $P^{m} \frac{1}{m} P^{0}$ on its domain

$$
D\left(T_{\tau}\right)=\left\{u \in H_{0}^{2}(D) \quad \mid \frac{1}{m}(\Delta+\tau) \in H^{2}(D)\right\}
$$

Proof.

We state without proof that $H_{0}^{2}(D)$ is dense in $L^{2}(D)$. To see that $t_{\tau}$ is semibounded, we write

$$
\begin{aligned}
t_{\tau}(u) & =\int_{D} \frac{1}{m}|(\Delta+\tau) u|^{2}+\tau \int_{D} \bar{u}((\Delta+\tau) u) \\
& \geq \frac{1}{\sup (m)}\|(\Delta+\tau) u\|^{2}-\tau\|(\Delta+\tau) u\|\|\mid u\| \\
& \geq\left(\frac{1}{\sup (m)}-\tau \varepsilon\right)\|(\Delta+\tau) u\|^{2}-\frac{\tau}{\varepsilon}\|u\|^{2} \\
& \geq \frac{1}{2 \sup (m)}\|(\Delta+\tau) u\|^{2}-2 \sup (m) \tau^{2}\|u\|^{2}
\end{aligned}
$$

after choosing $\varepsilon=\frac{1}{2 \tau \sup (m)}$. We record for later use the consequence of (5.7) that

$$
\|(\Delta+\tau) u\|^{2} \leq 2 \sup (m) t_{\tau}(u)+(2 \sup (m) \tau)^{2}\|u\|^{2}
$$

Every densely defined semi-bounded quadratic form defines a unique self-adjoint operator ([11], page 278), $T_{\tau}$, with domain the set of $u \in H_{0}^{2}(D)$ such that there is an $f \in L^{2}(D)$ with

$$
t(v, u)=(v, f)
$$

for all $v \in H_{0}^{2}(D)$, where $t(v, u)$ is the bilinear form,

$$
\begin{aligned}
t(v, u) & =\int_{D}\left(\overline{(\Delta+\tau) v} \frac{1}{m}(\Delta+\tau) u+\tau \bar{v}(\Delta+\tau) u\right. \\
& =\int_{D} \bar{v}\left((\Delta+\tau) \frac{1}{m}(\Delta+\tau)\right) u+\tau \int_{D} \bar{v}(\Delta+\tau) u \\
& =\left(v, T_{\tau} u\right)
\end{aligned}
$$


where the second and third equalities hold for all $u \in D\left(T_{\tau}\right)$, and illustrate that $T_{\tau}=P^{m} \frac{1}{m} P^{0}$ with $D\left(T_{\tau}\right)$ as asserted in (5.6).

LEMMA 5.3. $T_{\tau}$ has discrete spectrum which depends continuously on $\tau$.

Proof. Because $T_{\tau}$ is semi-bounded and $H_{0}^{2}(D)$ is compactly embedded in $L^{2}(D)$, $T_{\tau}$ has compact resolvent and therefore discrete spectrum. For $m$ 's that are not smooth, the domains of $T_{\tau}$ may depend $\tau$. We give a direct proof of the continuity of the eigenvalues. We shall show below that, for all positive real $\sigma$ and $\tau$,

$$
t_{\sigma}(u) \leq(1+M|\sigma-\tau|) t_{\tau}(u)+M\left(\tau^{2}+\sigma+1\right)|\sigma-\tau|\|u\|^{2}
$$

where the constant $M$ depends only on $m$. We recall the min-max characterization of the eigenvalues of a self-adjoint operator defined by a quadratic form (page 71 of [13])

$$
\lambda^{n}=\max _{W \in \mathcal{W}_{n}} \min _{\substack{u \in W \\\|u\|=1}} q(u)
$$

where $\mathcal{W}_{n}$ denotes the codimension $n$ subspaces of the form domain of $q$. An immediate consequence of (5.10) is that inequalities between quadratic forms imply the same inequalities for their ordered eigenvalues, so that (5.9) implies

$$
\lambda_{\sigma}^{n} \leq(1+M|\sigma-\tau|) \lambda_{\tau}^{n}+M\left(\tau^{2}+\sigma+1\right)|\sigma-\tau|
$$

and consequently,

$$
\lambda_{\sigma}^{n}-\lambda_{\tau}^{n} \leq|\sigma-\tau| M\left(\lambda_{\tau}^{n}+\left(\tau^{2}+\sigma+1\right)\right)
$$

Because we may interchange $\sigma$ and $\tau$,

$$
\left|\lambda_{\sigma}^{n}-\lambda_{\tau}^{n}\right| \leq|\sigma-\tau| M\left(\max \left(\lambda_{\tau}^{n}, \lambda_{\sigma}^{n}\right)+2\left(\tau^{2}+\sigma^{2}+1\right)\right)
$$

First fix $\sigma$ in (5.11) set $\tau=0$ to conclude that each $\lambda_{\sigma}^{n}$ varies only over a compact set when $\sigma$ varies over a compact set. Thus the maximum, $\max \left(\lambda_{\tau}^{n}, \lambda_{\sigma}^{n}\right)$, is bounded for $\sigma$ and $\tau$ on compact sets, and therefore (5.12) proves continuity of the eigenvalues.

It remains only to prove (5.9). We begin by writing

$$
\begin{aligned}
t_{\sigma}(u)-t_{\tau}(u)= & (\sigma-\tau) \int\left(\bar{u} \frac{1}{m}(\Delta+\tau) u+\overline{(\Delta+\tau) u} \frac{1}{m} u+\tau^{2}|u|^{2}\right) \\
& +(\sigma-\tau)^{2} \int\left(\bar{u}(\Delta+\tau) u+\frac{1}{m}|u|^{2}\right) \\
\leq & |\sigma-\tau|(1+|\sigma-\tau|) M\left(\|u\|\|(\Delta+\tau) u\|+\tau^{2}\|u\|^{2}\right)
\end{aligned}
$$

where $M$ depends only on $\frac{1}{m}$. For any $\varepsilon>0$

$$
\leq|\sigma-\tau|(1+|\sigma-\tau|) M\left(\varepsilon\|(\Delta+\tau) u\|^{2}+\left(\tau^{2}+\frac{1}{\varepsilon}\right)\|u\|^{2}\right)
$$

We make use of (5.8), to obtain with a different $M$

$$
\begin{aligned}
& \leq|\sigma-\tau|(1+|\sigma-\tau|) M\left(\varepsilon t_{\tau}(u)+\left((\varepsilon+1) \tau^{2}+\frac{1}{\varepsilon}\right)\|u\|^{2}\right) \\
& \leq|\sigma-\tau| M\left(t_{\tau}(u)+\left(\tau^{2}+\sigma+1\right) \|\left. u\right|^{2}\right)
\end{aligned}
$$

after choosing $\frac{1}{\varepsilon}=1+|\sigma-\tau|$. 
Lemma 5.4. If

$$
\operatorname{sign}(m) \inf _{u \in H_{0}^{2}(D)} \frac{t_{\tau}(u)}{\|u\|^{2}}>0
$$

then $\tau$ is not a transmission eigenvalue. If there exists $u \in H_{0}^{2}(D)$ such that

$$
\operatorname{sign}(m) \frac{t_{\tau}(u)}{\|u\|^{2}} \leq 0
$$

then there is a transmission eigenvalue $\tau^{*} \in[0, \tau]$.

Proof.

The hypothesis (5.13) implies that the spectrum of $T_{\tau}$ is strictly positive or strictly negative, hence it has no kernel.

The hypothesis (5.14) implies that $\operatorname{sign}(m) T_{\tau}$ has at least one non-positive eigenvalue. But $\operatorname{sign}(m) T_{0}$ is easily seen to be positive definite, so the lowest eigenvalue, which is a continuous function of $\tau$, must have passed through zero for some $\tau^{*} \in[0, \tau]$.

口

We will use a simple modification of Lemma 5.4 to show the existence of more than one transmission eigenvalue. We define the multiplicity of a transmission eigenvalue $\tau_{*}$ to be the multiplicity of 0 as an eigenvalue of $T_{\tau_{*}}$.

Lemma 5.5. If there exists a $\tau>0$ and a p-dimensional subspace $V^{p} \in H_{0}^{2}(D)$ such that

$$
\operatorname{sign}(m) \frac{t_{\tau}(u)}{\|u\|^{2}} \leq 0
$$

for all $u \in V^{p}$, then there are $p$ transmission eigenvalues, counting multiplicity, in $[0, \tau]$.

Proof. The hypothesis guarantees that $t_{\tau}$ has $p$ negative eigenvalues, counting multiplicity. The continuity of the spectrum implies that each of those eigenvalues must pass through zero as $\tau^{*}$ decreases from $\tau$ to 0 . Each time an eigenvalue passes through 0 , the dimension of the negative definite subspace, $V^{p}$, decreases by the multiplicity of the zero eigenvalue, so the sum of the multiplicities of the transmission eigenvalues between 0 and $\tau$ must be at least $p$. $\square$ We will need a few simple inequalities to prove the theorems to follow. We collect them in the lemma below.

LEMMA 5.6.

$$
\begin{gathered}
\lambda_{0}(D)=\inf _{u \in H_{0}^{1}(D)} \frac{\int_{D}|\operatorname{grad} u|^{2}}{\int_{D}|u|^{2}}=\inf _{u \in H_{0}^{2}(D)} \frac{\int_{D}|\operatorname{grad} u|^{2}}{\int_{D}|u|^{2}}>0 \\
\mu_{0}(D)=\inf _{u \in H_{0}^{2}(D)} \frac{\int_{D}|\Delta u|^{2}}{\int_{D}|u|^{2}} \geq \inf _{u \in H_{0}^{1} \cap H^{2}} \frac{\int_{D}|\Delta u|^{2}}{\int_{D}|u|^{2}}=\lambda_{0}(D)^{2}
\end{gathered}
$$

If $u \in H_{0}^{2}(D)$

$$
\lambda_{0}(D) \leq \frac{\int_{D}|\operatorname{grad} u|^{2}}{\int_{D}|u|^{2}} \leq \frac{\left(\int_{D}|\Delta u|^{2}\right)^{\frac{1}{2}}}{\left(\int_{D}|u|^{2}\right)^{\frac{1}{2}}}
$$


Proof. The first equality in (5.15) is the Rayleigh-Ritz characterization of the first Dirichlet eigenvalue. The second follows because $H_{0}^{2}(D)$ is dense in $H_{0}^{1}(D)$. The first equality in (5.16) is the Rayleigh-Ritz characterization of the the lowest eigenvalue of the biharmonic operator with Dirichlet boundary conditions, the lowest eigenvalue of the clamped plate. The inequality holds because $H_{0}^{2}(D) \subset H_{0}^{1}(D) \cap H^{2}(D)$, so the first infimum must be larger. The second infimum is exactly the Rayleigh-Ritz characterization for the lowest eigenvalue of the Dirichlet Laplacian squared ${ }^{6}$, which is the square of the first Dirichlet eigenvalue, which proves the final equality in (5.16). The first inequality in (5.17) follows from the meaning of infimum, and the second from integration by parts and the Cauchy-Schwartz inequality.

TheOREM 5.7. Suppose that $m>-1$ is a constant. If

$$
\tau \leq \min \left(1, \frac{1}{m+1}\right) \lambda_{0}(D)
$$

then $\tau$ is not a transmission eigenvalue. If

$$
\frac{\left(1+\frac{m}{2}\right)^{2}}{1+m} \geq \frac{\mu_{p}}{\lambda_{0}^{2}} \geq 1
$$

where $\mu_{p}(D)$ is the $(p+1)$ st clamped plate eigenvalue, then there are $p+1$ transmission eigenvalues $\tau$ with

$$
\tau \leq\left(\frac{m+2}{m+1}\right) \frac{\lambda_{0}(D)}{2}
$$

Proof. It follows from (5.4) that

$$
\begin{aligned}
m t_{\tau}(u) & =\|(\Delta+\tau(1+m)) u\|^{2}-m \tau \int_{D} \bar{u}(\Delta+\tau(1+m)) u \\
& >m \tau\left[\|\operatorname{grad} u\|^{2}-(1+m) \tau\|u\|^{2}\right] \\
& \geq m \tau\left[\lambda_{0}(D)-(1+m) \tau\right]\|u\|^{2}
\end{aligned}
$$

which shows that, for $0<m$ and $\tau \leq \frac{\lambda_{0}(D)}{1+m}$, $t_{\tau}$ is positive definite and therefore that $\tau$ is not a transmission eigenvalue. If $m<0$, we express $t_{\tau}$ as in (5.5)

$$
\begin{aligned}
m t_{\tau}(u) & =\|(\Delta+\tau) u\|^{2}-m \tau\|\operatorname{grad} u\|^{2}+m \tau^{2}\|u\|^{2} \\
& \geq(-m) \tau\|u\|^{2}\left(\lambda_{0}(D)-\tau\right)
\end{aligned}
$$

which shows that $t_{\tau}$ is positive definite as long as $\tau<\lambda_{0}(D)$, and finishes the proof of the assertion that $\tau$ satisfying (5.18) is not a transmission eigenvalue.

To prove the existence of transmission eigenvalues, we will use Lemma 5.5. Restricting to the sphere, $\|u\|^{2}=1$, we may write

\footnotetext{
${ }^{6}$ Functions in the domain of the square of the Dirichlet Laplacian must satisfy a second boundary condition, $\left.\Delta u\right|_{\partial D}=0$. Analogous to the case of the Neumann Laplacian, this is a free boundary condition, which doesn't appear explicitly in the definition of the form domain, and therefore not in the infimum in (5.16).
} 


$$
\begin{aligned}
m t_{\tau}(u) & =(m+1) \tau^{2}-2\left(1+\frac{m}{2}\right)\|\operatorname{grad} u\|^{2} \tau+\|\Delta u\|^{2} \\
& \leq(m+1) \tau^{2}-2\left(1+\frac{m}{2}\right) \lambda_{0} \tau+\|\Delta u\|^{2}
\end{aligned}
$$

We choose $\tau=\frac{1+\frac{m}{2}}{1+m} \lambda_{0}$ to obtain

$$
\leq-\frac{\left(1+\frac{m}{2}\right)^{2}}{1+m} \lambda_{0}^{2}+\|\Delta u\|^{2}
$$

and restrict $u$ to the eigenspace associated with the lowest $p+1$ clamped plate eigenvalues, so that

$$
m t_{\tau}(u) \leq-\frac{\left(1+\frac{m}{2}\right)^{2}}{1+m} \lambda_{0}^{2}+\mu_{p}
$$

Our hypothesis (5.19) is that this quantity is negative, so the conclusion (5.20) follows from Lemma 5.5.

THEOREM 5.8. Suppose that $m \in L^{\infty}(D)$. If

$$
\tau \leq \min \left(1, \frac{1}{\sup (m)+1}\right) \lambda_{0}(D)
$$

then $\tau$ is not a transmission eigenvalue. If $m>0$ and

$$
\inf (m) \geq 4 \frac{\mu_{p}^{\frac{1}{2}}}{\lambda_{0}}+\frac{\mu_{p}}{\lambda_{0}^{2}}
$$

there are $p+1$ transmission eigenvalues $\tau$ with

$$
\tau \leq \frac{\lambda_{0}(D)}{2}\left(\frac{\inf (m)-2 \frac{\mu_{p}^{\frac{1}{2}}}{\lambda_{0}}}{\inf (m)+1}\right)
$$

Proof. For $m>0$,

$$
\begin{aligned}
t_{\tau}(u) & =\int \frac{1}{m}|(\Delta+\tau(1+m)) u|^{2}-\tau \int \bar{u}(\Delta+\tau(1+m)) u \\
& \geq \tau\|\operatorname{grad} u\|^{2}-\tau^{2} \int(1+m)|u|^{2} \\
& \geq \tau\|u\|^{2}\left(\lambda_{0}-\tau(1+\sup m)\right)
\end{aligned}
$$

which shows that $t_{\tau}$ is positive definite if $\tau<\frac{\lambda_{0}}{1+\inf m}$ and therefore $\tau$ is not a transmission eigenvalue. For $m<0$

$$
\begin{aligned}
-t_{\tau}(u) & =\int \frac{1}{|m|}|(\Delta+\tau) u|^{2}-\tau \int \bar{u}(\Delta+\tau) u \\
& >\tau\|\operatorname{grad} u\|^{2}-\tau^{2}\|u\|^{2} \\
& \geq \tau\|u\|^{2}\left(\lambda_{0}-\tau\right)
\end{aligned}
$$

so that $-t_{\tau}$ is positive definite if $\tau<\lambda_{0}$, completing the proof of the first assertion. 
To prove existence, we write

$$
t_{\tau}(u)=\tau^{2} \int\left(1+\frac{1}{m}\right)|u|^{2}-\tau\left(\|\operatorname{grad} u\|^{2}+\int \frac{1}{m}(\bar{u} \Delta u+u \Delta \bar{u})\right)+\int \frac{1}{m}|\Delta u|^{2}
$$

We restrict to $\|u\|^{2}=1$ and write $S=\sup \left(\frac{1}{m}\right)$, to see that

$$
t_{\tau}(u) \leq \tau^{2}(1+S)-\tau\left(\|\operatorname{grad} u\|^{2}-2 S\|\Delta u\|\right)+S\|\Delta u\|^{2}
$$

Restricting to $V^{p}$ gives

$$
t_{\tau}(u) \leq \tau^{2}(1+S)-\tau\left(\lambda_{0}-2 S \mu_{p}^{\frac{1}{2}}\right)+S \mu_{p}
$$

We minimize the sum of the first two terms by choosing $\tau=\frac{\lambda_{0}-2 S \mu_{p}^{\frac{1}{2}}}{2(1+S)}$ to obtain

$$
\leq-\frac{\left(\lambda_{0}-2 S \mu_{p}^{\frac{1}{2}}\right)^{2}}{4(1+S)}+S \mu_{p}
$$

If we set $A=\frac{\lambda_{0}}{\mu_{p}^{\frac{1}{2}}}, t_{\tau}$ restricted to $V^{p}$ is non-positive if

$$
\begin{aligned}
(A-2 S)^{2}-4 S(1+S) & \geq 0 \\
A^{2}-4(A+1) S & \geq 0 \\
\frac{A^{2}}{4(A+1)} & \geq S
\end{aligned}
$$

which is equivalent to (5.21).

口

6. Conclusions. Under the hypothesis that the perturbation of the index of refraction is large enough, we have shown the existence of $D$-transmission eigenvalues, given upper and lower bounds for their locations, and identified the corresponding solutions to the transmission eigenvalue problem with the kernel of a scattering operator.

The upper and lower bounds for the transmission eigenvalues depend on the lowest eigenvalues of the Dirichlet Laplacian and the Dirichlet Bilaplacian (the clamped plate operator). These bounds show that the lowest transmission eigenvalue increases as the $L^{\infty}$ norm of $m$, or the size of its support, decreases. All of our bounds depend on $D$ only through these eigenvalues, and on $m$ only through its infimum or supremum. Thus our estimates for the transmission eigenvalues, $k^{2}$, scale with dilations just like the Dirichlet eigenvalues, as the reciprocal of the area of $D$.

In the Born, or weak scattering approximation, there are no transmission eigenvalues [7] when $m$ is strictly positive or strictly negative. Our results are consistent with this, but we don't know if there is a threshold below which there are no transmission eigenvalues, or the lowest transmission eigenvalue simply goes to infinity as $m$ decreases to zero. ${ }^{7}$

\footnotetext{
${ }^{7}$ See [3] for an inequality relating the lowest transmission eigenvalue (if it exists) to the supremum of $m$ and the diameter of $D$.
} 
In the radial case, there are infinitely many transmission eigenvalues, so it is reasonable to expect the same result here, but we have no results in this direction.

In summary, there are many questions remaining, some of which may be accessible by a further analysis of the quadratic forms of the operators introduced here. Because array imaging techniques are making the scattering operator, and hence its kernel, possible to measure, these questions are becoming increasingly relevant.

\section{REFERENCES}

[1] S. Agmon. Spectral properties of Schrödinger operators and scattering theory. Ann. Scuola Norm. Sup. Pisa Cl. Sci. (4), 2(2):151-218, 1975.

[2] S. Agmon and L. Hörmander. Asymptotic properties of solutions of differential equations with simple characteristics. J. Analyse Math., 30:1-38, 1976.

[3] F. Cakoni, D. Colton, and P. Monk. On the use of transmission eigenvalues to estimate the index of refraction from far field data. Inverse Problems, 23:507-522, 2007.

[4] D. Colton, A. Kirsch, and L. Päivärinta. Far-field patterns for acoustic waves in an inhomogeneous medium. SIAM J. Math. Anal., 20(6):1472-1483, 1989.

[5] D. Colton and R. Kress. Inverse acoustic and electromagnetic scattering theory, volume 93 of Applied Mathematical Sciences. Springer-Verlag, Berlin, second edition, 1998.

[6] D. Colton and P. Monk. The inverse scattering problem for acoustic waves in an inhomogeneous medium. In Inverse problems in partial differential equations (Arcata, CA, 1989), pages 73-84. SIAM, Philadelphia, PA, 1990.

[7] D. Colton, L. Päivärinta, and J. Sylvester. The interior transmission problem. Inverse Probl. Imaging, 1(1):13-28, 2007.

[8] L. Hörmander. The analysis of linear partial differential operators. II. Classics in Mathematics. Springer-Verlag, Berlin, 2005. Differential operators with constant coefficients, Reprint of the 1983 original.

[9] J. R. McLaughlin and P. L. Polyakov. On the uniqueness of a spherically symmetric speed of sound from transmission eigenvalues. J. Differential Equations, 107(2):351-382, 1994.

[10] J. R. McLaughlin, P. L. Polyakov, and P. E. Sacks. Reconstruction of a spherically symmetric speed of sound. SIAM J. Appl. Math., 54(5):1203-1223, 1994.

[11] M. Reed and B. Simon. Methods of modern mathematical physics. I. Academic Press Inc. [Harcourt Brace Jovanovich Publishers], New York, second edition, 1980. Functional analysis.

[12] B. P. Rynne and B. D. Sleeman. The interior transmission problem and inverse scattering from inhomogeneous media. SIAM J. Math. Anal., 22(6):1755-1762, 1991.

[13] B. Simon. Quantum mechanics for Hamiltonians defined as quadratic forms. Princeton University Press, Princeton, N. J., 1971. Princeton Series in Physics. 